\title{
História da Maternidade: em que ponto estamos? ${ }^{1}$
}

\author{
Anne Cova ${ }^{2}$
}

\begin{abstract}
Resumo
Este ensaio apresenta o que tem sido produzido sobre a maternidade pela historiografia. São, sobretudo, os trabalhos desenvolvidos na França, no campo da história das mulheres e dos estudos de gênero, que foram considerados, no momento de elaboração desse balanço historiográfico. No entanto, a contribuição de outras disciplinas para a elucidação do tema, especialmente da sociologia e da ciência política, e as pesquisas realizadas a respeito fora do território francês, particularmente em países de língua inglesa, não deixaram de ser abordadas. Trata-se de texto importante para os historiadores que se preocupam com a construção de uma história das mães ou da maternidade, o que justifica a sua tradução do francês para o português e a sua publicação no Brasil.
\end{abstract}

A história da maternidade é um tema vasto, que diz respeito a vários domínios à história das mulheres e do gênero, à história política, à história social, à história cultural -, mas também a diversas disciplinas - à sociologia e à ciência política, notadamente. O tema possui, então, forma imprecisa e não é possível, dentro dos limites deste artigo, abordá-lo a partir de todos os ângulos. Nós nos concentraremos sobre os trabalhos realizados na França em história das mulheres e do gênero, sem deixar de tentar esboçar algumas abordagens de outras disciplinas e evocar, sucintamente, as pesquisas desenvolvidas fora do território francês. ${ }^{3}$

\section{Da história das mães à história da proteção da maternidade}

Em história, a obra pioneira é a de Catherine Fouquet e de Yvonne Knibiehler, um estudo de longa duração: Histoire des mères du Moyen Âge à nos jours constitui uma obra de referência (FOUQUET; KNIBIEHLER, 1977). Continuando na pista desta

\footnotetext{
${ }^{1}$ Referência do original em francês: COVA, Anne. Où en est l'histoire de la maternité? Clio: Histoire, Femmes, Sociétés, Toulouse, n. 21, p.189-211, 2005.

2 Tradução de Marina Alves Amorim e Frederico Assis Cardoso.

${ }^{3}$ Dentre os balanços historiográficos em história das mulheres e do gênero, Cf. OFFEN; PIERSON; RENDALL (1991), THÉBAUD (1998), SOHN; THÉLAMON (1998); BOCK; COVA (2003).
} 
pesquisa, Yvonne Knibiehler publicou vários livros sobre a maternidade. La Révolution Maternelle analisa, através de três gerações de mulheres, como "a função materna organiza ou condiciona a cidadania das mulheres." (1997, p.12-13) Uma obra de síntese que trata da maternidade no vasto quadro geográfico do Ocidente (KNIBIEHLER, 2000). Nós não podemos retraçar a história das mães sem interpelar a história dos pais, porque les pères aussi ont une histoire [...] $]^{4}$ nos lembra a historiadora (KNIBIEHLER, 1987). Yvonne Knibiehler orientou, igualmente, estudos interdisciplinares sobre a maternidade, a fim de mostrar as diferentes perspectivas possíveis (KNIBIEHLER, 1999; 2001). Uma obra recente em homenagem a essa autora sublinha o quanto a maternidade é um eixo norteador de suas pesquisas e como Yvonne Knibiehler explorou todas as suas facetas (BERNOS; BITTON, 2004).

A medicalização durante o período entre as duas grandes guerras reteve a atenção de Françoise Thébaud, em Quand nos grand-mères donnaient la vie: la maternité en France dans l'entre-deux-guerres (THÉBAUD, 1986). Esse livro destaca um dos sentidos da palavra maternidade, que, como tantas outras, é polissêmica, e apresenta quatro acepções: a) é, ao mesmo tempo, um estado, isto quer dizer, a qualidade de mãe; b) uma função reprodutiva, a ação de carregar e de colocar crianças no mundo; c) uma obra de arte representando a mãe e o bebê; d) e um estabelecimento ou serviço hospitalar onde se fazem os partos (significado específico na língua francesa ${ }^{5}$ ). Françoise Thébaud insiste sobre a medicalização do nascimento e sobre o lugar onde acontecem os partos. Em uma história do parto e dos cuidados com o recémnascido, ela privilegia a obstetrícia e os progressos da puericultura e descreve, notadamente, a passagem do parto em casa para o parto no hospital.

Uma outra obra que privilegia a instituição maternidade, mas no século XIX, é a da americana Rachel G. Fuchs (1992), que desenvolveu um estudo meticuloso sobre uma amostra de aproximadamente 1500 mulheres que pariram, entre 1830 e 1900, na Maternidade de Paris. As possibilidades oferecidas às mulheres grávidas ou àquelas que já são mães são também o objeto principal do livro de Alisa Klaus (1993), que efetua uma comparação entre a França e os Estados Unidos, desde o fim do século XIX até os anos 1920. No coração dessa obra, figura a questão da mortalidade infantil, tema

\footnotetext{
${ }^{4}$ A autora lança mão do título de um livro - Les pères aussi ont une histoire (KNIBIEHLER, 1987) para compor a frase em que faz alusão a essa obra. Por isso a decisão de manter parte do texto em francês no corpo do artigo, deixando para a nota de rodapé a tradução para o português: os pais também têm uma história. (N.T.).

${ }^{5}$ Esse é um dos significados que palavra maternidade possui também em português. (N.T.).
} 
igualmente central no livro da demógrafa Catherine Rollet (1990), que faz uma análise fina das grandes leis de proteção da infância e de sua aplicação (da lei Roussel de 1874 àquela de 1904 sobre as crianças assistidas, notadamente).

A maternidade é um tema que ocupa um lugar particular na história dos feminismos. Os da primeira metade do século XX (ditos da primeira onda) tentaram, em numerosos países, servir-se da maternidade como trunfo para penetrar na esfera pública, reivindicando direitos para as mães e para as mulheres em geral (COVA, 1997). ${ }^{6}$ Ao contrário, os feminismos da segunda onda criticaram a maternidade, considerada como um freio da emancipação das mulheres (FORTINO, 1997). As feministas da primeira onda não pararam de reclamar leis em favor da proteção da maternidade. Estudar a gênese da proteção da maternidade, que mistura a incitação e a repressão, e cobre todas as disposições que o Estado adotou no que concerne às mães, é estudar um aspecto essencial das políticas demográfica e social. Também foi sublinhada a diversidade das situações: mãe operária ou trabalhadora, dona de casa, mãe de muitos filhos ou não, mãe cujos filhos faleceram, mãe solteira, casada, divorciada ou viúva, mãe jovem ou em idade avançada, etc.

Desde o fim do século XIX, o tema da "despopulação" é recorrente entre os parlamentares, que acusam a queda da natalidade e reclamam a proteção da maternidade. Karen Offen (1984) analisou a relação entre despopulação, nacionalismo e feminismo. O movimento a favor do crescimento da natalidade atinge seu apogeu na França, como mostraram os trabalhos do historiador inglês Peter Tomlinson (1984), de Françoise Thébaud (1985)e de dois historiadores americanos, Cheryl Koos (1996; 1999) e Joshua Cole (2000). Essas pesquisas enfatizaram um grupo a favor do crescimento da natalidade, a Alliance Nationale pour l'Accroissement de la Population Française, que simboliza o movimento a favor do crescimento da natalidade francês, que, em comparação com outros países europeus, é "o mais forte na classe política, como na cultura popular tradicional” (BOCK, 1992a, p. 904)

A historiadora Molly Ladd-Taylor (1993) sublinha que, nos Estados Unidos, o medo da "despopulação" não faz sentido, mas que, para o período 1890-1930, movimentos de mulheres muito heterogêneos se baseiam na retórica da maternidade. Da mesma maneira, na França, ao longo da Terceira República, grupos de mulheres, não necessariamente feministas, tal a maioria de associações de mulheres católicas, reivindicam medidas em favor das mães (COVA, 2000). Numerosos manuais

\footnotetext{
${ }^{6}$ Sobre a Alemanha (1800-1914), Cf. ALLEN (1991).
} 
devocionais destinados às mulheres cristãs descrevem suas "missões sociais", seu “dever social”, que é a maternidade (LÉVY, 1984). O lugar considerável que ocupa as associações de mulheres na ação social foi colocado em evidência por Evelyne Diébolt (DIÉBOLT, 2001). Um grupo de mulheres católicas francesas, a Union Féminine Civique et Sociale (UFCS), fundada em 1925, que tentou, notadamente, impulsionar a legislação a favor das ajudas sociais voltadas para a família, reteve a atenção da cientista política Naomi Black (1989)e das historiadoras Susan Pedersen (1993) e Laura Lee Downs (2002). A UFCS levou a cabo, durante o período entre as duas grandes guerras, na França, uma ardente campanha a favor do retorno das mães ao lar. O slogan "o lugar da mulher é em casa" se propagou também na Alemanha nazista, onde ele se refere ainda a uma "casa" entendida como o país inteiro. Mas, como mostrou Gisela Bock (1992b), o regime é contra o crescimento da natalidade, já que é unicamente para uma minoria de mães que é instaurado um culto da maternidade - aliás, nenhuma lei é colocada em favor das mães, entre 1933 e 1942 (BOCK, 1992b, p. 155). ${ }^{7}$ Foi também estudado, no caso da Alemanha nazista, o tratamento diferente infligido às mães, segundo a geração à qual elas pertencem (HEINEMAN, 2001; 2003). Na Itália fascista, o depósito de ajudas sociais ligadas à maternidade por certas empresas foi analisado por Perry Willson (1993). A proteção da maternidade na Alemanha, depois de 1945, é o objeto da obra de Robert Moeller (1993). ${ }^{8}$

A proteção da maternidade não pode ser bem compreendida sem evocar a questão do trabalho fora de casa, que divide o movimento das mulheres. As medidas de proteção da maternidade são, às vezes, ressentidas como um recuo, mesmo se a grande maioria do movimento feminista francês da primeira onda se declara a favor das licenças maternidade. Os efeitos perversos da proteção do trabalho das mulheres foram analisados, no que concerne à França de 1879 a 1919, por Mary Lynn Stewart (1989) e Marilyn J. Boxer (1986). A legislação protetora pode, de fato, ter como consequência marginalizar as mulheres da população ativa e encorajar sua exploração pelo viés do trabalho no domicílio.

Uma das numerosas contribuições da história das mulheres e do gênero é estabelecer que as fronteiras são móveis entre o privado e o público e que não há duas esferas distintas. Função privada por excelência, a maternidade foi utilizada por numerosas feministas do passado para penetrar no espaço público e obter novos direitos

\footnotetext{
${ }^{7}$ Cf. também BOCK (1991).

${ }^{8}$ Cf. também CARPENTER (2001).
} 
(notadamente o direito de voto), que argumentaram que as aptidões das mães para gerir a receita familiar lhes permitiriam se ocupar das finanças do Estado e reivindicaram a maternidade como uma função social. Esse processo questiona o modelo de cidadania pensado por T.H. Marshall (1964), baseado na ideia de um progresso linear em três séculos: direitos civis adquiridos no século XVIII, direitos políticos adquiridos no século XIX, direitos sociais adquiridos no século XX. De fato, ele não é aplicável às mulheres que, na maioria dos países industrializados, obtiveram os direitos civis, políticos e sociais somente no século XX. Ele não é tampouco generalizável a todos os países, já que na Alemanha, por exemplo, direitos sociais foram adquiridos antes do sufrágio dito universal. Nos Estados Unidos, igualmente, Theda Skocpol (1992) constata que as leis voltadas para as mães são promulgadas em muitos Estados, desde os anos 1910, antes que todas as americanas se beneficiem do direito de voto. Quanto à França, mesmo sem mencionar o direito de voto, as mulheres casadas só obtiveram sua capacidade civil em 1938, enquanto as primeiras leis sociais de proteção da maternidade datam de 1909 e 1913. A teoria de T. H. Marshall suscitou numerosos comentários críticos, dentre eles o de David Held (1989, p. 199), que lhe reprova de não levar em conta, notadamente, questões colocadas pelas feministas, e o de Linda Gordon (1990b, p. 18), que o acusa de ignorar a história das relações das mulheres com o Estado e de não contestar a dependência das mulheres frente aos salários dos homens.

Da mesma maneira que foi questionado "o gênero da nação" (AUSLANDER; ZANCARINI-FOURNEL, 2000), os trabalhos sobre o Estado de bem-estar social diferem no que diz respeito ao que eles entendem por Estado. Douglas E. Ashford (1976), na sua obra The Emergence of Empire States, centrada, sobretudo, na Inglaterra e na França, sublinha que, nesse último País, a legislação social é parte integrante do desenvolvimento do Estado (p. 79-81). Isso não significa que o Estado seja exclusivamente sensível às pressões externas; ele possui uma autonomia própria. Autonomia que a abordagem dita neoinstitucionalista (state-centered approach) - à qual Theda Skocpol associou o seu nome - coloca no centro de seu propósito (EVANS; RUESCHEMEYER; SKOCPOL, 1990). Em Protecting Soldiers and Mothers: the political origins of social policy in the United States, ela se recusa a conceder um lugar muito grande ao determinismo burocrático e propõe uma polity centered-analysis. Linda Gordon lhe reprova, todavia, por negligenciar a atividade não-governamental, o papel do movimento operário e das ideologias e, de uma forma geral, de excluir a sociedade 
de suas explicações. Desse debate, ${ }^{9}$ o mais interessante é que todas duas concordam sobre a importância dos movimentos de mulheres na construção do Estado de bem-estar social. De fato, suas problemáticas se completam, combinando as prerrogativas da intervenção estatal com a importância do papel dos diferentes atores e atrizes da sociedade e das redes.

Vários temas adjacentes à maternidade foram objeto de estudos, tais como a profissionalização do trabalho das nutrizes (FÄY-SALLOIS, 1980; SUSSMAN, 1982; FILDES, 1988; CLARK, 2000; SCHULTHEISS, 2001) e das enfermeiras (KNIBIEHLER et al; 1984; COHEN, 2000), as questões ligadas ao corpo e à sexualidade, ${ }^{10}$ as teorias sobre o matriarcado (ALLEN, 1999). Prática antiga e conhecida em numerosos países, o recurso às amas-de-leite atinge, na França, no século XIX, picos em nenhuma parte alcançados na Europa. Uma questão justaposta ao recurso às amas-de-leite é a do abandono de crianças, o que concerne em média aproximadamente 25000 crianças por ano na França, no século XIX. Suas mães são urbanas, frequentemente solteiras, com idade média de vinte anos e, em quase um terço dos casos, domésticas. Abandonos e recurso às amas-de-leite diminuem no fim do século XIX, para se tornar raros no século XX. Mais precisamente, o recurso às amasde-leite diminui com a lei Roussel, a pasteurização do leite e o aumento das creches, mas continua sendo uma alternativa que perdura até o grande declínio, depois de 1914 (FUCHS, 1983; TAEGER, 1989; LAPLAIGE, 1989).

Em um grande número de discursos, a anatomia do corpo das mulheres as predestina à maternidade. Os seios, as ancas generosas para receber o feto, não deixam pairar nenhuma dúvida: a mulher é concebida para ser mãe. A "natureza” decidiu assim, e, daí a deduzir que a mulher deve se consagrar exclusivamente à maternidade, há somente um passo, que ultrapassam alegremente os médicos, cujas proposições foram objeto de múltiplos trabalhos (KNIBIEHLER, 1976; FOUQUET; KNIBIEHLER, 1983; CAROL, 2003). O corpo das mulheres grávidas suscita múltiplos conselhos, de higiene notadamente. Se certos médicos, tal como Adolphe Pinard, são progressistas, o corpo médico no seu conjunto é conservador e a velha sentença tota mulier in útero serve para lembrá-lo. O discurso médico propaga a ideia que o cérebro feminino é influenciado pelo útero, reduzindo a mulher a um sexo consagrado à reprodução. A maioria do corpo

\footnotetext{
${ }^{9}$ Sobre esse debate, Cf. GORDON (1993).

${ }^{10}$ Ver o balanço construído por Chaperon (2002) sobre a história contemporânea das sexualidades na França.
} 
médico se pronuncia contra o aborto e se regozija quando são promulgadas na França as leis de 1920-1923 que condenam o aborto, reprimem a propaganda anticoncepcional e oprimem os neomalthusianos (GAUTHIER, 2002; ZANCARINI-FOURNEL, 2002; LE NAOUR; VALENTI, 2003; GUERRAND, 1971; RONSIN, 1980). A França não é a única a adotar medidas repressivas. Na Itália, leis de pubblica sicurezza de 1926 são destinadas a impedir a propaganda em favor do aborto e do controle dos nascimentos. $\mathrm{O}$ aborto transforma-se em um crime de Estado, a venda de anticoncepcionais é proibida, assim como a educação sexual, mas essas leis são votadas por um Estado fascista (DETRAGIACHE, 1980; SARACENO, 1991, 2003; GRAZIA, 1992; GIORGIO, 1992; PICKERING-LAZZI, 1995; D’AMELIA, 1997; QUINE, 2002). Na França, a condenação de uma "fazedora de anjos" à guilhotina por Vichy coloca a questão da continuidade ou não do regime (Limore Yagil/ Francine Müel-Dreyfus), enquanto outros trabalhos insistiram sobre a importância do código da família de 1939, demonstrando a especificidade dos anos negros (Michèle Bordeaux, Miranda Pollard) (MÜEL-DREYFUS, 1996; YAGIL, 1997; POLLARD, 1998; BORDEAUX, 2002). Na Alemanha, a República de Weimar adota, igualmente, uma legislação repressiva em matéria de aborto: o artigo 218 faz do aborto um ato criminoso e prevê penas de encarceramento para uma mulher que aborta e para todo aquele que a ajuda; a propaganda anticoncepcional é também proibida (GROSSMANN, 1978).

É também a partir da Alemanha que se difundem as teorias sobre o matriarcado, notadamente com Das Mutterrecht (1861) de Bachofen, que mostra que o matriarcado precedeu o patriarcado com um período de ginecocracia. Essas teorias conhecem uma posteridade entre certas feministas, na Europa e nos Estados Unidos, de 1860 a 1914 (ALLEN, 1999). Enquanto o mito do matriarcado segue sendo o apanágio de uma minoria de feministas radicais, o mito das mães pacifistas "por natureza" beneficia, por sua vez, de um largo crédito junto à maioria das feministas. A sueca Ellen Key (18491926), cujo impacto é particularmente importante nos países anglo-saxões e na Alemanha, é conhecida igualmente na França como uma teorizadora da maternidade. Figura controversa no seu tempo e ainda hoje, ela estabelece uma ponte entre pacifismo e maternidade (PIERSON, 1990), glorificando a maternidade e a ternura maternal na sua obra War, Peace and the Future (1916). Segundo ela, o laço das mulheres com a guerra e a paz é diferente do dos homens, porque elas colocam crianças no mundo. Os romances publicados no período entre as duas grandes guerras, tal como Madame ne veut pas d'enfant, foram estudados por Mary Louise Roberts (ROBERTS, 1994). 
Considerando o que foi dito até agora, surge a necessidade de periodizar. No seu estudo comparativo França/ Inglaterra sobre a maternidade durante a Grande Guerra que proclama que a maternidade é um dever patriótico para as mulheres e coloca com mais acuidade do que anteriormente a questão da conciliação do trabalho com a maternidade -, Suzan Grayzel sublinha a importância da cronologia e insiste sobre a necessidade de escrever uma história cultural comparada da guerra (GRAYZEL, 1999; 2004). Isso nos conduz a evocar os trabalhos transnacionais ou comparativos, que se multiplicaram depois de uma quinzena de anos e constituem sempre uma linha de pesquisa promissora.

\section{Por uma história comparada dos Estados de Bem-Estar Social que leva em conta a categoria gênero}

Depois da publicação, em 1991, da obra precursora de Gisela Bock e de Pat Thane sobre mulheres e Estados de bem-estar social, os estudos floresceram nesse domínio. Dentre as contribuições inovadoras dessa obra figuravam a introdução do gênero e o fato de levar em conta o papel dos movimentos de mulheres no estudo da gênese do Estado de bem-estar social. Esse livro ilustrava também a pertinência da abordagem comparativa, analisando a situação na Alemanha (Gisela Bock e Irene Stoehr); Inglaterra (Jane Lewis e Pat Thane); Espanha (Mary Nash); França (Anne Cova e Karen Offen); Itália (Amarita Buttafuoco e Chiara Saraceno); Noruega (Ida Blom, Anne-Lise Seip e Hilde Ibsen); e Suécia (Ann-Sofie Ohlander). Os trabalhos que seguiram não estão todos de acordo sobre seus efeitos de gênero: alguns deles consideram que o Estado de bem-estar reforça a desigualdade, enquanto outros privilegiam os aspectos reformistas. Da mesma forma, a expressão "feminismo maternalista”, empregada por Gisela Bock (1992a), Seth Koven e Sonya Michel (1993) - transformar a maternidade função privada em uma função pública -, foi retomada por numerosos(as) historiadores(as), mas o que ela encobre deu lugar a definições diferentes.

Susan Pedersen argumenta que o modelo do "male breadwinner" - a "família ideal" é dirigida por um homem assalariado e comporta uma mulher dependente e crianças - é característico da Inglaterra, enquanto, na França, prevalece o "parental welfare state", modelo onde não está colocado como premissa que as mulheres são necessariamente dependentes, nem que os homens têm sempre famílias para sustentar 
(PEDERSEN, 1993). Essa pesquisa concede também um lugar importante à análise das ajudas sociais voltadas para a família, onde a França (lei de 1932) é pioneira. ${ }^{11}$ A Inglaterra só instaura ajudas sociais voltadas para a família (family allowances) depois da Segunda Guerra Mundial (1945), a Alemanha em 1935 (kinderbeihilfen), a Itália em 1936 (assegni familiari), a Espanha em 1938 (subsidio familiar), Portugal em 1942 (abono de família), a Noruega em 1946 (barnetrygd), a Suécia em 1947 (allmänna barnbidrag) e a Dinamarca em 1952 (bornetilskud). A expressão Estado de bem-estar social é atestada, na França, desde o Segundo Império, a fim de designar, ao mesmo tempo, uma desconfiança frente à intervenção crescente do Estado e uma crítica de um individualismo excessivo. Na Inglaterra, o termo Welfare State, que significa literalmente "Estado do bem-estar", só surge nos anos 1940. Mas o que ele abarca começou bem antes. Todavia, alguns pesquisadores preferem utilizar antes o termo social policy que Welfare State, evocador demais do pós-Segunda Guerra Mundial.

São, sobretudo, os(as) historiadores(as) anglo-americanos(as) que se prenderam à reflexão sobre a problemática mulheres e Estado de bem-estar social, tal qual Jane Lewis que escreveu e coordenou numerosas publicações (LEWIS, 1980; 1983; 1991; 1996; 1998; 2002). A historiadora inglesa Pat Thane (1991, 1991a) analisou, em uma perspectiva de gênero, a proteção da maternidade na Grã Bretanha (1860-1918). Elinor Accampo, Rachel Fuchs e Mary Lynn Stewart organizaram uma obra coletiva sobre as mulheres e Estado de bem-estar social na França (1870-1914) (ACCAMPO; FUCHS; STEWART, 1995). Mirian Cohen e Michel Hanagan (1991) publicaram um artigo comparativo centrado no período 1900-1940. A destacar, igualmente, o papel da revista americana Social Politics, International Studies in Gender, State \& Society, cujo primeiro número tem, agora, dez anos de existência. Isso ilustra bem que a questão é relativamente recente e que ela continua sendo, sobretudo, o apanágio dos trabalhos anglo-americanos e dos trabalhos universitários feministas. De fato, em um balanço dos trabalhos feministas sobre o Welfare State, que Linda Gordon construiu em Woman, The State, and Welfare de 1990, ela não cita nenhuma obra, nem mesmo nenhum artigo francês (GORDON, 1990b). Numerosos review articles publicados desde então concordam sobre a necessidade de efetuar uma abordagem de gênero do Estado de bemestar social como realizada na obra de Haney (1998) Engendering the Welfare State.

\footnotetext{
${ }^{11}$ Sobre as ajudas sociais voltadas para a família e a comparação França/ Inglaterra em uma perspectiva de gênero Cf. também MISRA (1998).
} 
Assim, os estudos comparativos se multiplicaram, quer se trate de comparar o modelo francês com a Inglaterra (Susan Pedersen, Susan Grayzel e Laura Lee Downs) ou de colocar em perspectiva o caso francês e seu homólogo americano (Alisa Klaus). O modelo americano (notadamente o Sheppard-Towner Act de 1921 sobre a maternidade e a infância) serve também de referência e suscita trabalhos comparativos com outras legislações, tal qual a em vigor na Nova Zelândia durante a primeira metade do século XX (BRYDER, 2003). Se a maior parte dos trabalhos comparativos se concentra sobre dois países, alguns efetuam uma análise tripartida: Inglaterra/Estados Unidos/França (1830-1995); Argentina/Estados Unidos/França (1880-1920), para mencionar dois exemplos (AUSLANDER; ZANCARINI-FOURNEL, 1995; MEAD, 2000).

Nos Estados Unidos, dentre as problemáticas que mais retiveram a atenção das historiadoras, figura a situação das mães solteiras, estudadas por Linda Gordon em um livro que é referência, mas também por Regina Kunzel e Gwendolyn Mink (GORDON, 1994; ${ }^{12}$ KUNZEL, 1993; MINK, 1995). Essas obras sublinham as diferenças entre as condições de vida das mães solteiras negras e as de suas homólogas brancas. Na mesma ordem de ideias, Molly Ladd-Taylor (1993) menciona que as políticas voltadas para a maternidade eram políticas raciais, no sentido em que elas concerniam unicamente às mulheres brancas. Essa preocupação de introduzir a noção de "raça" é umas das características dos trabalhos americanos em história das mulheres e do gênero. Em uma obra coletiva organizada por Molly Ladd-Taylor e Lauri Umansky (1997), são analisadas as repreensões às quais estão expostas as "más" mães e mais particularmente as mães solteiras, acusadas de todos os males.

No Canadá, igualmente, vários trabalhos se concentraram sobre a maternidade e, em particular, sobre as mães solteiras no século XX (COMACCHIO, 1993; MURRAY, 2004). Na Austrália, Marilyn Lake (1993)se prendeu à reflexão, em uma perspectiva histórica, sobre a maternidade e suas incidências sobre a cidadania. Uma obra comparativa organizada por Valérie Fildes, Lara Marks e Hilary Marland (1992) trata da situação das mães das antigas colônias e ambiciona abarcar cinco continentes, com o estudo do Canadá, da Austrália, da África do Sul, da Birmânia e da Malásia para o período 1870-1945.

Dentre as obras de síntese, individuais ou coletivas, que concedem um lugar à maternidade em uma perspectiva transnacional, figura a de Gisela Bock (2002) sobre as mulheres na história europeia, da Idade Média aos nossos dias. A maternidade está no

\footnotetext{
${ }^{12}$ Cf. também GORDON (1991; 1992a; 1992b).
} 
cerne de numerosos capítulos do livro de Karen Offen (2000) consagrado aos feminismos europeus de 1700 a 1950. O volume dedicado ao século XX de História das Mulheres no Ocidente, organizado por Françoise Thébaud (1995), comporta vários artigos sobre a maternidade e os Estados de bem-estar social. A Encyclopédie Politique et Historique des Femmes: Europe, Amérique du Nord, organizada por Christine Fauré (1997), aborda, igualmente, em vários momentos a maternidade. Enfim, a obra coletiva recente Le Siècle des Féminismes propõe dois capítulos sobre esse tema: "La Maternité, un enjeu dans le premier XXe siècle" e "Notre corps, nous-mêmes" (GUBIN, 2004).

Para fechar esse sobrevôo, mencionemos as contribuições pioneiras de outras disciplinas e, em particular, da sociologia e da ciência política. Assim, Jane Jenson realizou, desde os anos 1980, vários trabalhos comparativos França/ Estados Unidos (JENSON, 1986; 1987; 1989). Ela organizou, junto com a cientista política Mariette Sineau, uma obra com o título sugestivo Qui doit garder le jeune enfant? Modes d'accueil et travail des mères dans l'Europe en crise (JENSON; SINEAU, 1998). ${ }^{13} \mathrm{O}$ Welfare State contemporâneo e suas incidências sobre o trabalho das mães foi objeto de várias pesquisas, quer se trate de analisar as atividades profissionais das mães de família na França (1942-1982), como fez Jacqueline Martin; de compará-la com a situação em outros países (Gornick, Meyers, Ross; Morgan); de sublinhar a importância do modelo escandinavo (Ruggie; Arnlaug; Sainsbury) (MARTIN, 1998 ; GORNICK ; MEYERS ; ROSS, 1997 ; MORGAN, 2003 ; RUGGIE, 1984 ; ARNLAUG, 1992 ; SAINSBURY, 1999, 2001). Uma cientista política, a italiana Alisa del Re (1994), convida a não considerar as políticas sociais como gender neutral. Em uma obra organizada por duas sociólogas, Arlette Gautier e Jacqueline Heinen (1993), é analisado o papel no Estado de bem-estar dos movimentos feministas contemporâneos em cinco países (França, GrãBretanha, Itália, Polônia e ex-RDA - República Democrática Alemã). As mudanças ocorridas na ex-RDA e nos países do leste europeu foram objeto recentemente de pesquisas em história, sociologia e ciência política, três disciplinas que ocupam um papel chave na análise comparativa histórica em ciências sociais (GERHARD, 2001; ALSOP; HOCKEY, 2001; FODOR et al., 2002; HANEY, 2002). Este método de pesquisa se encontra, atualmente, no coração das ciências sociais, como o mostrou uma obra publicada em 2003, organizada por um cientista político e um sociólogo americanos, James Mahoney e Dietrich Rueschemeyer (2003). Em tal quadro

\footnotetext{
${ }^{13}$ Cf. também JENSON; SINEAU (2001).
} 
conceitual, os estudos sobre o gênero constituem, segundo Theda Skocpol (2003, p. 421), one of the most exciting new frontiers in comparative historical scholarship ${ }^{14}$. Essa idéia se aplica, igualmente, à história das mulheres e do gênero, sendo que Susan Pedersen (2004) sublinha, a justo título, as suas afinidades com o método comparativo.

A guisa de conclusão, inesgotáveis parecem ser os temas que gravitam em torno da maternidade em diversas disciplinas. Na hora do balanço, nós reteremos, em função de todos esses trabalhos, que a história da maternidade alcançou incontestavelmente uma maturidade. Da história das mães à história da proteção da maternidade, até uma história comparada da maternidade e dos Estados de bem-estar social que leva em conta a categoria gênero, nós medimos o caminho percorrido. Cruzar o gênero e o Estado de bem-estar social continua parecendo uma linha forte de pesquisa. É igualmente uma história comparativa que se vislumbra com os últimos trabalhos e anuncia belos dias para esse domínio de pesquisa.

\section{Referências}

ACCAMPO, Elinor; FUCHS, Rachel G.; STEWART, Mary Lynn (Org.). Gender and the politics of social reform in France (1870-1914). Baltimore: The John Hopkins University Press, 1995.

ALLEN, Ann Taylor. Feminism and motherhood in Germany (1800-1914). New Brunswick: Rutgers University Press, 1991.

ALLEN, Ann Taylor. Feminism, social science and the meanings of modernity: the debate on the origin of the family in Europe and the United States (1860-1914). American Historical Review, p. 1085-1113, Oct. 1999.

ALSOP, Rachel; HOCKEY, Jenny. Women's reproductive lives as a symbolic resource in Central and Eastern Europe. The european journal of women's studies, v. 8, n. 4, p. 454-471, 2001.

ARNLAUG, Leira. Welfare states and working mothers: the scandinavian experience. Cambridge: Cambridge University Press, 1992.

\footnotetext{
14 “[...] uma das novas fronteiras mais excitantes na erudição histórica comparativa.” (N.T.)
} 
ARNUP, Katherine; LEVESQUE, Andrée; PIERSON, Ruth Roach (Org.). Delivering motherhood: maternal ideologies and practices in the $19^{\text {th }}$ and $20^{\text {th }}$ centuries. New York: Routledge, 1990.

ASHFORD, Douglas E. The emergence of the welfare states. Oxford: Basil Blackwell, 1986.

AUSLANDER, Leora; ZANCARINI-FOURNEL, Michelle (Org.). Différence des sexes et protection sociale (XIXe-XXe siècles). Paris: Presses Universitaires de Vincennes, 1995.

AUSLANDER, Leora; ZANCARINI-FOURNEL, Michelle. Le genre de la nation. Clio, histoire, femmes et sociétés, Toulouse, n. 12, 2000.

BERNOS, Marcel; BITTON, Michèle (Org.). Femmes, familles, filiations: société et histoire. Aix-en-Provence : Presses de l’Université de Provence, 2004.

BLACK, Naomi. Social feminism. Ithaca: Cornell University Press, 1989.

BLOM, Ida. Voluntary motherhood (1900-1930): theories and politics of a norwegian feminist in an international perspective. In: BOCK, Gisela; THANE, Pat (Org.). Maternity and gender policies: women and the rise of the European welfare states, (1880s-1950s). New York: Routledge, 1991. p. 21-39.

BOCK, Gisela; THANE, Pat (Org.). Maternity and gender policies: women and the rise of the european welfare states, (1880s-1950s). New York: Routledge, 1991.

BOCK, Gisela. Antinatalism, maternity and paternity in the national socialist racism. In: BOCK, Gisela; THANE, Pat (Org.). Maternity and gender policies: women and the rise of the European welfare states, (1880s-1950s). New York: Routledge, 1991. p. 233255.

BOCK, Gisela. Pobreza feminina, maternidade e direitos das mães na ascensão dos Estados-providência (1890-1950). In: THÉBAUD, Françoise (Org.). História das mulheres no ocidente: o século XX. Coleção organizada por Georges Duby e Michèle Perrot. Porto: Edições Afrontamento, 1992. p. 435-477. (História das mulheres no ocidente).

BOCK, Gisela. A política sexual nacional-socialista e a história das mulheres. In: THÉBAUD, Françoise (Org.). História das mulheres no ocidente: o século XX. Coleção organizada por Georges Duby e Michèle Perrot. Porto: Edições Afrontamento, 1992. p. 185-219. (História das mulheres no ocidente).

BOCK, Gisela. Women in european history. Oxford: Blackwell, 2002.

BOCK, Gisela; COVA, Anne (Org.). Writing women's history in Southern Europe $\left(1^{\text {th }}-\mathbf{- 2 0}^{\text {th }}\right.$ Centuries). Écrire l'histoire des femmes en Europe du Sud (XIXe-XXe siècles). Oeiras: Celta, 2003. 
BORDEAUX, Michèle. La victoire de la famille dans la France Défaite (Vichy, 1940-1944). Paris: Flammarion, 2002.

BOXER, Marilyn J. Protective legislation and home industry: the marginalization of women workers in late nineteenth-early twentieth century France. Journal of social history, p. 45-65, 1986.

BRYDER, Linda. Two models of infant welfare in the first half of the Twentieth Century: New Zealand and the USA. Women's history review, v.12, n. 4, p. 547-558, 2003.

BUTTAFUOCO, Annarita. Motherwood as a political strategy: the role of the italian women's moviment in the creation of the Cassa Nazionale di Maternità. In: BOCK, Gisela; THANE, Pat (Org.). Maternity and gender policies: women and the rise of the european welfare states, (1880s-1950s). New York: Routledge, 1991. p. 178-195.

CAROL, Anne. Le genre face aux mutations du savoir médical: sexes et nature féminine dans la fécondation (XVIe-XIXe siècles). In: CAPDEVILA, Luc et al. Le genre face aux mutations: masculin et féminin du Moyen Âge à nos jours. Rennes: Presses Universitaires de Rennes, 2003. p. 83-92.

CARPENTER, K. M. N. For mothers only: mothers convalescent homes and modernizing maternal ideology in 1950s West Germany. Journal of Social History, v. 34, n. 4, p. 863-893, 2001.

CHAPERON, Sylvie. L'histoire contemporaine des sexualités en France. Vingtième Siècle, 75, p. 47-59, jul./set. 2002.

CLARK, Linda. The rise of professional women in France: gender and public administration since 1830. Cambridge: Cambridge University Press, 2000.

COHEN, Miriam; HANAGAN, Michael. The politics of gender and the making of the welfare state (1900-1940): a comparative perspective. Journal of Social History, v. 24, n. 3, p. 469-484, 1991.

COHEN, Yolande. Profession infirmière: une histoire des soins infirmiers dans les hôpitaux du Québec. Montréal: Presses de l’Université de Montréal, 2000.

COLE, Joshua. The power of large numbers: population, politics and gender in nineteenth-century France. Ithaca: Cornell University Press, 2000.

COMACCHIO, Cynthia R. Nations are built of babies: saving Ontario's mothers and children (1900-1940). Toronto: Toronto University Press, 1993.

COVA, Anne. French feminism and maternity: theories and policies (1880-1918). In: BOCK, Gisela; THANE, Pat (Org.). Maternity and gender policies: women and the rise of the european welfare states, (1880s-1950s). New York: Routledge, 1991. p. 119137. 
COVA, Anne. Maternité et droits des femmes en France (XIXe-XXe siècles). Paris: Anthropos-Economica, 1997.

COVA, Anne. Au service de l'eglise, de la patrie et de la famille. In: Femmes catholiques et maternité sous la IIIe République. Paris: L’Harmattan, 2000.

COVA, Anne. La maternité : un enjeu dans le premier XXe siècle. In: GUBIN, Eliane et al. (Org.). Le siècle des féminismes. Paris: Les Éditions de l'Atelier, 2004. p. 195-208.

D’AMELIA, Marina (Org.). Storia della maternità. Roma: Laterza, 1997.

DETRAGIACHE, Denise. Un aspect de la politique démographique de l'Italie fasciste: la répression de l'avortement. Mélanges de l'ecole française de Rome, v. 92, 1980.

DIEBOLT, Evelyne. Les femmes dans l'action sanitaire, sociale et culturelle (19012001): les associations face aux institutions. Paris: Femmes et Associations, 2001.

DOWNS, Laura Lee. L'inégalité à la Chaîne: la division sexuée du travail dans l'industrie métallurgique en France et en Angleterre (1914-1939). Paris: Albin Michel, 2002.

EVANS, Peter B.; RUBSCHEMEYER, Dietrich; SKOCPOL, Theda (Org.). Bringing the state back in. Cambridge: Cambridge University Press, 1990. ( $1^{\mathrm{a}}$ ed.: 1985).

FAURE, Christine (Org.). Encyclopédie politique et historique des femmes: Europe, Amérique du Nord. Paris: PUF, 1997.

FAY-SALLOIS, Fanny. Les nourrices à Paris au XIXe siècle. Paris: Payot, 1980.

FILDES, Valerie. Wet nursing: a history from antiquity to the present. Oxford: Blackwell, 1988.

FILDES, Valerie; Marks, Lara; MARLAND, Hilary (Org.). Women and children first: international maternal and infant welfare (1870-1945). London: Routledge, 1992.

FODOR, Eva; GLASS, Christy; KAWACHI, Janette; POPESCU, Livia. Family policies and gender in Hungary, Poland and Romania. Communist and PostCommunist studies, v. 35, n. 4, p. 475-490, Dec. 2002.

FORTINO, Sabine. De filles en mères: la seconde vague du féminisme et la maternité. Clio, histoire, femmes et sociétés, Toulouse, n. 5, p. 217-238, 1997.

FOUQUET, Catherine; KNIBIEHLER, Yvonne. Histoire des mères du Moyen Âge à nos jours. Paris: Montalba, 1977.

FOUQUET, Catherine; KNIBIEHLER, Yvonne. La femme et les médecins: analyse historique. Paris: Hachette, 1983.

FUCHS, Rachel G. Abandoned children: foundlings and child welfare in nineteenth century France. Albany: University of New York Press, 1983. 
FUCHS, Rachel G. Poor and pregnant in Paris: strategies for survival in the nineteenth century. New Jersey: Rutgers University Press, 1992.

GAUTHIER, Xavière. Naissance d'une liberté. Contraception, avortement: le grand combat des femmes au XXe siècle. Paris: Robert Laffont, 2002.

GAUTIER, Arlette; HEINEN, Jacqueline (Org.). Le sexe des politiques sociales. Paris: Côté-femmes, 1993.

GERHARD, Ute. Politique sociale et maternité: le cas de l'allemagne à l'est et à l'ouest. Travail, genre et société, n. 6, p.59-81, 2001.

GIORGIO, Michela De. Le italiane dall'unità ad oggi. Roma: Laterza, 1992.

GORDON, Linda (Org.). Women, the state and welfare. Madison: The University of Wisconsin Press, 1990.

GORDON, Linda. The new feminist scholarship on the welfare state. In: GORDON, Linda (Org.). Women, the state and welfare. Madison: The University of Wisconsin Press, 1990. p. 9-35.

GORDON, Linda. Black and white of welfare: women's welfare activism (1890-1945). The Journal of American History, v. 78, n. 2, p. 559-590, Sep. 1991.

GORDON, Linda. Why Nineteenth-century feminists did not support "Birth Control" and Twentieth-Century feminists do: feminism, reproduction and the family. In: THORNE, Barrie; YALOM, Marilyn (Org.). Rethinking the family: some feminist questions. Boston: Northeastern University Press, 1992. p. 140-154.

GORDON, Linda. Social insurance and public assistance: the influence of gender in welfare thought in the United States (1890-1935). American historical review, v. 97, n. 1, p. 19-54, Feb. 1992.

GORDON, Linda. Gender, State and society: a debate with Theda Skocpol. Contention, v. 2, n. 3, p. 139-156, Spring, 1993.

GORDON, Linda. Pitied but not entitled: single mothers and the history of welfare. New York: Free Press, 1994.

GORNICK, Janet C.; MEYERS, Marcia K., ROSS, Katherin E. Supporting the employment of mothers: policy variation across fourteen welfare states. Journal of european social policy, v. 7, n. 1, p. 45-70, 1997.

GRAYZEL, Susan R. Women's identities at war: gender, motherhood and politics in Britain and France during the First World War. Chapel Hill: The University of North Carolina Press, 1999. 
GRAYZEL, Susan R. Across battle fronts: gender and the comparative cultural history of modern european war. In: COHEN, Deborah; O'CONNOR, Maura (Org.). Comparison and history: Europe in cross-national perspective. London: Routledge, 2004. p. 71-84.

GRAZIA, Victoria de. How fascism ruled woman Italy (1922-1945). Berkeley: University of California Press, 1992.

GROSSMANN, Atina. Abortion and economic crisis: the 1931 campaign against 218 in Germany. New german critique, v. 14, p. 119-137, Spring, 1978.

GUBIN, Eliane et al. (Org.). Le siècle des féminismes. Paris: Les Éditions de l'Atelier, 2004.

GUERRAND, Roger-Henri. La libre maternité (1896-1969). Tournai: Casterman, 1971.

HANEY, Lynne A. Engendering the welfare state: a review article. Comparative studies in society and history, v. 40, n. 4, p. 748-767, 1998.

HANEY, Lynne A. Inventing the needy: gender and the politics of welfare in Hungary. Berkeley: University of California Press, 2002.

HEINEMAN, Elizabeth D. Age and generation in women's history. Whose mothers? Generational difference, war and the nazi cult motherhood. Journal of Women's History, v. 12, n. 4, p. 138-163, 2001.

HEINEMAN, Elizabeth D. What difference does a husband make? Women and marital status in Nazi and Postwar Germany. Berkeley: University of California Press, 2003. ( $1^{\text {a }}$ ed.: 1999).

HELD, David. Political theory and the modern state: essays on state, power and democracy. Stanford: Stanford University Press, 1989.

IBSEN, Hilde; SHIP, Anne-Lise. Family Welfare, Which policy? Norway's road to child allowances. In: BOCK, Gisela; THANE, Pat (Org.). Maternity and gender policies: women and the rise of the European welfare states, (1880s-1950s). New York: Routledge, 1991. p. 40-59.

JENSON, Jane. Gender and reproduction: or, babies and the state. Studies in political economy, v. 20, p.9-45, Summer, 1986.

JENSON, Jane. Both friend and foe: woman and state welfare. In: BRIDENTHAL, Renate; KOONZ, Claudia; STUARD, Susan (Org.). Becoming visible: women in European history. Boston: Houghton Mifflin, 1987. p. 535-556. $\left(1^{\mathrm{a}}\right.$ ed.: 1977)

JENSON, Jane. Paradigms and political discourse: protective legislation in France and the United States before 1914. Canadian journal of political science, v. XXII, n. 2, p. 235-258, Jun. 1989. 
JENSON, Jane; SINEAU, Mariette (Org.). Qui doit garder le jeune enfant? Modes d'accueil et travail des mères dans l'Europe en crise. Paris: LGDJ, 1998.

JENSON, Jane; SINEAU, Mariette (Org.). Who cares? Women's work, childcare and welfare state redesign. Toronto: Toronto University Press, 2001.

KERTZER, David I.; BARBAGLI, Marzio (Org.). The history of the european family: family life in the twentieth century. New Haven: Yale University Press, 2003.

KLAUS, Alisa. Every child a lion: the origins of maternal and infant health policy in the United States and France (1890-1920). Ithaca: Cornell University Press, 1993.

KNIBIEHLER, Yvonne. Les médecins et la "nature féminine" au temps du code civil. Annales, n. 4, p. 824-845, jul./ago. 1976.

KNIBIEHLER, Yvonne et al. Cornettes et Blouses Blanches: les infirmières dans la société française (1880-1980). Paris: Hachette, 1984.

KNIBIEHLER, Yvonne. Les pères aussi ont une histoire... Paris: Hachette, 1987.

KNIBIEHLER, Yvonne. La révolution maternelle: femmes, maternité, citoyenneté depuis 1945. Paris: Perrin, 1997.

KNIBIEHLER, Yvonne (Org.). Repenser la maternité. Panoramiques, n. 40, 1999.

KNIBIEHLER, Yvonne. Histoire des mères et de la maternité en occident. Paris: PUF, 2000.

KNIBIEHLER, Yvonne (Org.). Maternité: affaire privé, affaire publique. Paris: Bayard, 2001.

KOOS, Cheryl Ann. Engendering reaction: the politics of pronatalism and the family in France (1919-1944). 1996. Tese (Doutorado) - University of Southern California, California.

KOOS, Cheryl Ann. On les aura! The gendered politics of abortion and the Alliance Nationale contre la Depopulation (1938-1944). Modern and contemporary France, v.7, n. 1, p. 21-34, fev. 1999.

KOVEN, Seth; MICHEL, Sonya (Org.). Mothers of a new world: maternalist politics and the origins of welfare states. London: Routledge, 1993.

KUNZEL, Regina. Fallen women, problem girls: unmarried mothers and the professionalization of social work (1890-1945). New Haven: Yale University Press, 1993.

LADD-TAYLOR, Molly. Mother, work, women, child welfare and the state (18901930). University of Illinois Press, 1993. 
LADD-TAYLOR, Molly; UMANSKY, Lauri (Org.). "Bad" mothers: the politics of blame in twentieth-century America. New York: New York University Press, 1997.

LAKE, Marilyn. A revolution in the family: the challenge and contradictions of maternal citizenship in Australia. In: KOVEN, Seth; MICHEL, Sonya (Org.). Mothers of a new world: maternalist politics and the origins of welfare states. London: Routledge, 1993. p.378-395.

LAPLAIGE, Danielle. Sans famille à Paris: orphelins et enfants abandonnés de la seine au XIXe siècle. Paris: Centurion, 1989.

LE NAOUR, Jean-Yves; VALENTI, Catherine. Histoire de l'avortement (XIXe-XXe siècle). Paris: Seuil, 2003.

LEVY, Marie-Françoise. De mères en filles: l'éducation des françaises (1850-1880). Paris: Calmann-Lévy, 1984.

LEWIS, Jane. The Politics of Motherhood: child and maternal welfare in England (1900-1939). London: Croom Helrn, 1980.

LEWIS, Jane (Org.). Women's welfare, women's rights. London: Croom \& Helrn, 1983.

LEWIS, Jane. Models of equality for women: the case of state support for children in twentieth-century Britain. In: BOCK, Gisela; THANE, Pat (Org.). Maternity and gender policies: women and the rise of the European welfare states, (1880s-1950s). New York: Routledge, 1991. p. 73-92.

LEWIS, Jane. Gender and welfare in the late nineteenth and early twentieth century. In: DIGBY, Anne; STEWART, John (Org.). Gender, health and welfare. London: Routledge, 1996. p. 208-228.

LEWIS, Jane (Org.). Gender, social care and welfare state restructuring in Europe. Aldershot: Ashgate, 1998.

LEWIS, Jane. Gender and Welfare State Change. European Societies, v. 4, n. 4, p. 331-357, 2002.

MAHONEY, James; RUESCHEMEYER, Dietrich (Org.). Comparative historical analysis in the social sciences. Cambridge: Cambridge University Press, 2003.

MARSHALL, T. H. Class, citizenship and social development. New York: Doubleday \& Company, 1964.

MARTIN, Jacqueline. Politique Familiale et Travail des Femmes Mariées en France: perspective historique (1942-1982). Population, v. 6, p. 1119-1152, 1998.

MEAD, Karen. Beneficent maternalism: argentine motherhood in comparative perspective (1880-1920). Journal of women's wistory, v. 12, n. 3, p. 120-145, Autumn, 2000. 
MINK, Gwendolyn. The wages of motherhood: inequality in the Welfare State (19171942). Ithaca: Cornell University Press, 1995.

MISRA, Joya. Mothers or workers? The value of women's labor: women and the emergence of family allowance policy. Gender and society, v. 12, n. 4, p.376-399, ago. 1998.

MOELLER, Robert G. Protecting motherhood: women and the family in the politics of postwar West Germany. Berkeley: University of California Press, 1993.

MORGAN, Kimberly J. The politics of mothers' employment: France in comparative perspective. World politics, v. 55, p.259-289, jan. 2003.

MUEL-DRYFUS, Francine. Vichy et l'eternel féminin: contribution à une sociologie politique de l'ordre des corps. Paris: Seuil, 1996.

MURRAY, Karen Bridget. Governing « unwed mothers » in Toronto at the turn of the Twentieth Century. The canadian historical review, v. 85, n. 2, p.253-276, jun. 2004.

NASH, Mary. Pronatalism and motherhood in franco's Spain. In: BOCK, Gisela; THANE, Pat (Org.). Maternity and gender policies: women and the rise of the European welfare states, (1880s-1950s). New York: Routledge, 1991. p. 160-177.

OFFEN, Karen. Depopulation, nacionalism and feminism in Fin-de-Siècle France. American historical review, v. 89, n. 3, p. 648-676, jun. 1984.

OFFEN, Karen; PIERSON, Ruth Roach; RENDALL, Jane (Org.). Writing women's history: international perspectives. Bloomington: Indiana University Press, 1991.

OFFEN, Karen. Body politics: women, work and the politics of motherhood in France (1920-1950). In: BOCK, Gisela; THANE, Pat (Org.). Maternity and gender policies: women and the rise of the European welfare states, (1880s-1950s). New York: Routledge, 1991. p. 138-159.

OFFEN, Karen. European feminism (1700-1950): a political history. Stanford: Stanford University Press, 2000.

OHLANDER, Ann-Sofie. The invisible child? The struggle for a social democratic family policy in Sweden (1900-1960). In: BOCK, Gisela; THANE, Pat (Org.). Maternity and gender policies: women and the rise of the European welfare states, (1880s-1950s). New York: Routledge, 1991. p. 60-72.

PEDERSEN, Susan. Family dependence and the origins of the welfare state: Britain and France (1914-1945). Cambridge: Cambridge University Press, 1993.

PEDERSEN, Susan. Comparative history and women's history: explaining convergence and divergence. In: COHEN, Deborah; O'CONNOR, Maura (Org.). Comparison and history: Europe in cross-national perspective. London: Routledge, 2004. p. 85-102. 
PICKERING-LAZZI, Robin (Org.). Mothers of Invention: women, Italian fascism and culture. Minneapolis: University of Minnesota Press, 1995.

PIERSON, Ruth Roach. Ellen Key: maternalism and pacifism. In: ARNUP, Katherine; LEVESQUE, Andrée; PIERSON, Ruth Roach (Org.). Delivering Motherhood: maternal ideologies and practices in the $19^{\text {th }}$ and $20^{\text {th }}$ centuries. New York: Routledge, 1990. p. 270-283.

POLLARD, Miranda. Reign of virtue: mobilizing gender in Vichy France. Chicago: Chicago University Press, 1998.

QUINE, Maria Sophia. Italy's social revolution: charity and welfare from liberalism to fascism. New York: Palgrave, 2002.

RE, Alisa Del. Les femmes et l'État-providence: les politiques sociales en France dans les années trente. Paris: L'Harmattan, 1994.

ROBERTS, Mary Louise. Civilization without sexes: reconstructing gender in postwar France (1917-1927). Chicago: The University of Chicago Press, 1994.

ROLLET-ÉCHALIER, Catherine. La politique à l'Égard de la petite enfance sous la IIIe République. Paris: INED, 1990. (Travaux et Documents, 127).

RONSIN, Francis. La grève des ventres: propagande néo-malthusienne et baisse de la natalité française (XIXe-XXe siècles). Paris: Aubier Montaigne, 1980.

RUGGIE, Mary. The state and working women: a comparative study of Britain and Sweden. Princeton: Princeton University Press, 1984.

SAINSBURY, Diane (Org.). Gender and welfare state regimes. Oxford: Oxford University Press, 1999.

SAINSBURY, Diane. Gender and the making of welfare states: Norway and Sweden. Social politics: international studies in gender, state \& society, v. 8, p.113-143, 2001.

SARACENO, Chiara. Redefining maternity and paternity: gender, pronatalism and social policies in fascist Italy. In: BOCK, Gisela; THANE, Pat (Org.). Maternity and gender policies: women and the rise of the European welfare states, (1880s-1950s). New York: Routledge, 1991. p. 196-212.

SARACENO, Chiara. Social and family policy. In: KERTZER, David I.; BARBAGLI, Marzio (Org.). The history of the european family: family life in the twentieth century. New Haven: Yale University Press, 2003. p.238-269.

SCHULTHEISS, Katrin. Bodies and souls: politics and professionalization of nursing in France (1880-1922). Cambridge: Harvard University Press, 2001.

SKOCPOL, Theda. Protecting soldiers and mothers: the political origins of social policy in the United States. Cambridge: Harvard University Press, 1992. 
SKOCPOL, Theda. Doubly engaged social science: the promise of comparative historical analysis. In: MAHONEY, James; RUESCHEMEYER, Dietrich (Org.). Comparative historical analysis in the social sciences. Cambridge: Cambridge University Press, 2003. p. 407-428.

SOHN, Anne-Marie; THELAMON, Françoise (Org.). L'histoire sans les femmes estelle possible? Paris: Perrin, 1998.

STEWART, Mary Lynn. Women, work and the french state: labour protection and social patriarchy (1879-1919). Kingston: McGill-Queen's University Press, 1989.

STOEHR, Irene. Housework and motherhood: debates and politics in the women's movement in Imperial Germany and the Weimar Republic. In: BOCK, Gisela; THANE, Pat (Org.) Maternity and gender policies: women and the rise of the European welfare states, (1880s-1950s). New York: Routledge, 1991. p. 213-232.

SUSSMAN, George D. Selling mothers' milk: the wet-nursing business in France (1715-1914). Urbana: University of Illinois Press, 1982.

TAEGER, Angela. L'état, les enfants trouvés et les allocations familiales en France (XIXe-XXe siècles). Francia, p. 15-33, 1989.

THANE, Pat. Genre et protection sociale: la protection maternelle et infantile en Grande-Bretagne (1860-1918). Genèses, n. 6, p. 73-97, dez. 1991.

THANE, Pat. Visions of gender in the making of the british welfare state: the case of women in the british labour party and social policy (1906-1945). In: BOCK, Gisela; THANE, Pat (Org.). Maternity and gender policies: women and the rise of the European welfare states, (1880s-1950s). New York: Routledge, 1991. p. 93-118.

THÉBAUD, Françoise. Le movement nataliste dans la France de l'Entre-Deux-Guerres: L'Alliance Nationale pour l'accroissement de la population Française. Revue d'histoire moderne et contemporaine, v. 32, p. 276-301, abr./jun. 1985.

THÉBAUD, Françoise. Quand nos grand-mères donnaient la vie: la maternité en France dans l'entre-deux-guerres. Lyon: PUL, 1986.

THÉBAUD, Françoise (Org.). História das mulheres no ocidente: o século XX. Coleção organizada por Georges Duby e Michèle Perrot. Porto: Edições Afrontamento, 1992. (História das Mulheres no Ocidente).

THÉBAUD, Françoise. Écrire l'histoire des femmes. Fontenay/Saint-Cloud: ENS, 1998.

THOLINSON, Richard Peter. The politics of dénatalité during the french Third Republic (1890-1940). 1984. Tese (Doutorado) - Christ's College, Cambridge.

WILLSON, Perry R. The clockwork factory: women and work in fascist Italy. Oxford: Clarendon Press, 1993. 
YAGIL, Limore. La politique familiale de Vichy et la conception de la "femme nouvelle”. Guerres mondiales et conflits contemporains, n. 188, p. 27-49. 1997.

ZANCARINI-FOURNEL, Michelle. Genre et politique: les années 1968. Vingtième Siècle, 75, p.133-143, jul./set. 2002.

ZANCARINI-FOURNEL, Michelle. Notre Corps, Nous-mêmes. In: GUBIN, Eliane et al. (Org.). Le siècle des féminismes. Paris: Les Éditions de l'Atelier, 2004. p.209-220. 